This is the submitted version of the article:

Portela A., Calvo-Lozano O., Estevez M., Medina Escuela A., Lechuga L. M.. Optical nanogap antennas as plasmonic biosensors for the detection of miRNA biomarkers. J ournal of Materials Chemistry B, (2020). 8. : 4310 - . 10.1039/d0tb00307g.

Available at: https://dx.doi.org/10.1039/d0tb00307g 


\section{Optical nanogap antennas as plasmonic biosensors for the detection of miRNA biomarkers}

Received 00th January 20xx, Accepted 00th January 20xx DOI: $10.1039 / \times 0 \times x 00000 x$

\author{
Alejandro Portela, Olalla Calvo-Lozano, M.-Carmen Estevez*, Laura M. Lechuga
}

\begin{abstract}
Nanoplasmonic biosensors based on nanogap antennas structures usually demand complex and expensive fabrication processes in order to achieve a good performance and sensitve detection. We here report the fabrication of large-area nanoplasmonic sensor chips based on nanogap antennas by employing a customized, simple and low-cost colloidal lithography process. By precisely controlling the angle for tilted e-beam metal evaporation, an elliptical mask is produced, which defines the total length of the dipole antenna nanostructures while assuring that the plasmonic response is oriented in the same direction along the sensor chip. Large-area sensor chips of nanogap antennas formed by pairs of gold nanodisks separated by gaps with an average size of $11.6 \pm 4.7 \mathrm{~nm}$ are obtained. The optical characterization of the nanogap antenna structures in an attenuated total reflection (ATR) configuration shows a bulk refractive index sensitivity of $422 \mathrm{~nm} / \mathrm{RIU}$, which is in agreement with FDTD numerical simulations. The biosensing potential of the $\mathrm{cm}^{2}$-sized nanostructured plasmonic sensor chips has been evaluated for the detection of miRNA-210, a relevant biomarker for lung

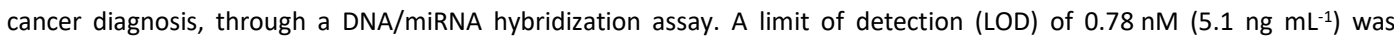
achieved with no need of further amplification steps, demonstrating the high sensitivity of these plasmonic nanogap antennas for the direct and label-free detection of low molecular weight biomolecules as miRNAs.
\end{abstract}

\section{Introduction}

Several disciplines related to biology, pharmaceutics and medicine require powerful biosensing tools for the study of biomolecular interactions or for the detection and validation of new biomarkers. To fulfil these demands, biosensor devices with high sensitivity, competitive limit of detections (LOD) and high robustness and reproducibility are needed. Furthermore, ideally the sensing method should require a minimum sample pre-treatment, should be easy-to-use, and highly reliable. For all the above, optical biosensors are highly ranked among the best choice for advanced biosensor platforms. Thanks to the progress on the last decade in the field of biophotonics, labelfree biosensors have received tremendous momentum achieving competitive results for the ultrasensitive detection of very small analytes at extremely low concentrations with no need of labelling. ${ }^{1,2}$ This introduces several advantages since a label-free configuration leads to simplified sample manipulation (skipping laborious steps required for adding label tags), and it avoids possible interferences from the biomolecule tagging.

Plasmonic sensing exploiting the intrinsic sensitivity of resonant coupling of optical waves with the free electron cloud

\footnotetext{
a. Nanobiosensors and Bioanalytical Applications Group (NanoB2A), Catalan Institute of Nanoscience and Nanotechnology (ICN2), CSIC, CIBER-BBN and BIST, Campus UAB Bellaterra, 08193 Barcelona, Spain.
}

of noble metals have been widely studied.3.4 Plasmonic biosensors have demonstrated their full potential for monitoring molecular recognition events by detecting the refractive index (RI) variations occurring in the close vicinity of the plasmonic metal surface. Particularly, sensors based on localized surface plasmon resonance (LSPR), excited on subwavelength nanostructures are powerful configurations with demonstrated excellent performance and great potential for integration and multiplexed analysis. Besides conventional designs (i.e nanoparticles, nanorods, nanopillars, etc), there are plenty of examples of LSPR sensor elements obtained after precisely engineering nanostructures using different materials, geometry, and arrangements (i.e. single, ordered, nonordered). ${ }^{5-8}$ This is done usually aiming for enhanced sensitivity, by mainly addressing the generation of areas with local field enhancement. LSPR sensing has led to the detection of small analytes such as short peptides chains, DNA fragments, small biomolecules, etc., deposited in a very low concentration (even a single molecule level) on the interface of the plasmonics features. ${ }^{9-12}$

Plasmonic gold nanogap antennas (i.e two nanostructures separated by a nanogap of few nanometers) are nanostructures supporting the confinement of a high local electromagnetic field within the nanoscale gap formed by the arms of a dipole antenna. ${ }^{13}$ The close proximity of the metallic nanoparticles (NPs) induces the plasmon coupling between them, leading to the formation of new hybrid plasmon modes that shown a strong field enhancement. ${ }^{14}$ Some applications of 
these structures have already demonstrated their capabilities for plasmonic trapping ${ }^{15,16}$ and in surface enhancement of fluorescence and Raman spectroscopy. ${ }^{17,18}$ These effects are remarkably observed on the visible light frequencies for gap sizes below $20 \mathrm{~nm}$, which represents a fabrication challenge when a large-area sensing surface is required. ${ }^{19,20}$ Most nanogap antennas structures explored for biosensing applications have been fabricated through expensive and timeconsuming fabrication methods such as e-beam lithography ${ }^{19}$ or focus ion beam (FIB), ${ }^{21}$ which hinders their further transfer into more feasible and commercial-oriented products.

In this work we demonstrate the performance of a plasmonic biosensor based on optical nanogap antennas with average gap size of $\approx 12 \mathrm{~nm}$ that are fabricated using a low cost and high throughput nanofabrication process. Through numerical EM field simulations, we confirm the improvement on sensitivity for gap sizes below $15 \mathrm{~nm}$, which is attributed to the condition of the EM field generated. The fabrication protocol for obtaining large surface chips with these nanostructures and its flexibility for the modification of geometries (gap sizes and length) has been also evaluated. This is supported by the experimental characterization of the fabricated nanogap antennas, including the assessment of their bulk sensitivity. A complementary hybridization DNA/RNA assay for a relevant lung cancer biomarker (miRNA-210) is selected as an experimental demonstration of the capabilities of the optical nanogap antenna biosensor for direct and label-free biodetection of low molecular weight analytes.

\section{Experimental}

\section{Nanolithography process and SEM characterization}

Nanoplasmonic chips of randomly distributed arrays of gold nanogap antennas (two nanodisks of variable shape and diameter, height $=20 \mathrm{~nm}$, separated by a nanogap of $12 \mathrm{~nm}$ ) were fabricated on glass substrates following a modified protocol of the colloidal hole-mask lithography $(\mathrm{HCL})$ process as described before. ${ }^{22}$ Thorough cleaning process of glass slides was performed using SDS (sodium dodecyl sulfate), $\mathrm{HCl}$, and Milli-Q water and drying under $\mathrm{N}_{2}$ flow. Then, the sensor chips were sonicated for $5 \mathrm{~min}$ at $50{ }^{\circ} \mathrm{C}$, first in acetone and then in isopropanol, and finally dried under $\mathrm{N}_{2}$ flow. Surface silanization of the glass substrate was performed by immersing the chip overnight in a solution of isobutyl (trimethoxy)silane (10 $\mu \mathrm{L}$ of in $5 \mathrm{~mL}$ toluene) to improve the coating of a PMMA (polymethyl methacrylate $950 \mathrm{~K}$ ) layer. The glass substrate was then rinsed with toluene and isopropanol and dried under $\mathrm{N}_{2}$ flow. A thin layer of PMMA diluted at $4 \%$ in anisole (thickness about $210 \mathrm{~nm}$ ), was spin-coated (4000 rpm, $1500 \mathrm{r} \mathrm{s}^{-2}$ ), onto the glass-treated substrate, and immediately baked at $165^{\circ} \mathrm{C}$ during $3 \mathrm{~min}$. A short $\mathrm{O}_{2}$ plasma treatment $(10 \mathrm{~s}$, Power $=75$ $\mathrm{W}$, Pressure $=75 \mathrm{mTorr}$ ) was applied to increase the hydrophilicity of the PMMA layer. This was followed by a drop- coating onto the PMMA layer of a cationic solution (PDDA, (poly (diallyldimethylammonium) chloride, $0.2 \mathrm{wt} \%$, in $\mathrm{H}_{2} \mathrm{O}$ ) during $1 \mathrm{~min}$, then rinsing with Milli-Q water and dried under $\mathrm{N}_{2}$ flow. A solution of nanoparticles (NPs) (sulfate latex beads, $80 \mathrm{~nm}$ diameter; FisherScientific, Spain) is diluted in water (0.01 wt\%) and sonicated for 5 min prior to drop-coating (1 $\mathrm{min}$ ) on the glass chip. The chip was then immersed in water $\left(95^{\circ} \mathrm{C}, 3 \mathrm{~min}\right.$ ) to fix the NPs on the PMMA layer prior to rinse again with Milli-Q water and dry under $\mathrm{N}_{2}$ flow.

The evaporation of a Titanium (Ti) sacrificial layer of $20 \mathrm{~nm}$ thickness using an e-gun evaporator (EB273, Telemark, Germany) allowed producing a round (i.e. normal $90^{\circ}$ evaporation) or an elliptical shape mask (i.e. by tilting the sample stage respect to the normal $90^{\circ}$ evaporation). A tapestripping was conducted to remove the NPs, leaving the Tihole mask with ellipsoidal shape features that exposes the PMMA. Selective etching of the thin PMMA layer was achieved using a reactive ion etcher (RIE) (Oxford PlasmaPro ${ }^{\circledR} 100$ Cobra Oxford Instruments, UK) using $\mathrm{O}_{2}$ plasma under the following conditions: 5 min RIE $=400 \mathrm{~W}$, Pressure $=75 \mathrm{mTorr}, \mathrm{O}_{2}$-flux $=50$ sccm). The PMMA etched on the surrounding volume under the ellipsoidal-shaped holes leaves a small cavity underneath the $\mathrm{Ti}$ layer where the glass substrate is exposed and accessible for the Au evaporation.

Tilted metal e-beam evaporation of $\mathrm{Au} 19 \mathrm{~nm}$ is conducted two times, using equal values of opposite angles respect to the normal evaporation. In order to improve the Au adhesion to the glass surface, the evaporation of each Au nanodisk forming the dimer is preceded by a deposition of a thin Ti layer $(1 \mathrm{~nm})$ using the same angles. In this way, the Ti/Au layers are deposited at each side of the nanoholes. A lift-off process is conducted by first rinsing in acetone followed by three short cycles ( $<5 \mathrm{~s}$ ) of sonication in acetone in order to detach any remaining $\mathrm{Au}$ and $\mathrm{Ti}$ coated on top of the PMMA layer. The sensor chips were rinsed using isopropanol and dried under $\mathrm{N}_{2}$ flow.

\section{Numerical Simulations}

Near field analysis and far field spectra of single nanodisks (80 $\mathrm{nm}$ diameter) and nanogap antennas ( $80 \mathrm{~nm}$ diameter each arm separated by a $15 \mathrm{~nm}$ gap) were obtained using a 3D finite-difference time-domain method (FDTD), (FDTD Solutions, Lumerical Inc., Canada). The plasmonic features are placed on top of a $\mathrm{SiO}_{2}$ surface and excited using a broadband light (550-900 nm), choosing a plane wave illumination source incident from the substrate. The light is linearly polarized parallel (in-plane) to the Au features. For the case of nanogap antennas, the electric field vector is set parallel (longitudinal) to the axis of the nanogap. In all cases a variable size rectangular mesh, with an initial $2 \mathrm{~nm}$ mesh, was defined on the vicinity of the Au features $(40 \mathrm{~nm}$, where near-field produced by the localized plasmon resonance is certainly confined). Boundary conditions are set as perfectly matched 
layer (PML). Dielectric permittivity of $\mathrm{Au}$ is selected using standard values from Johnson \& Christy. ${ }^{23}$

\section{Optical setup and bulk sensitivity characterization}

An optical setup has been configured using a fibered broadband light source (Thorlabs, SLS201L). The fiber's end is set into an angle adjustable optomechanical support, together with a collimator lens and a linear polarizer. The beam is normally incident on the facet of a BK-7 trapezoidal prism with an $80^{\circ}$ base angle. The sensor chip is placed onto the prism using matching oil, and both are further clamped to a microfluidic cell where the plasmonic nanostructures are exposed to the liquid sample solutions. The light reflected from the sensor chip is then focused into an optical fiber connected to a USB spectrophotometer (FLAME-S-VIS-NIR-ES, Ocean Optics) controlled by an in-house software program, which tracks and plots the LSPR peak location in real-time.

A syringe pump is employed for the flow delivery of the samples. An injection valve incorporating a loop $(200 \mu \mathrm{L}$ volume) connected to both the pump and the inlet of the microfluidic cell was employed to deliver the samples to the microfluidic cell. The evaluation of the bulk sensitivity of the sensor chips was performed as follows: Milli-Q water was set under continuous flow ( $\left.35 \mu \mathrm{L} \cdot \mathrm{min}^{-1}\right)$ and solutions with known values of $\mathrm{RI}$ (glycerol solutions at different concentrations) were sequentially injected into the sensor surface, registering the changes of the plasmonic spectra over time and tracking the shift of the LSPR extinction peak $(\Delta \lambda)$.

\section{Surface biofunctionalization and DNA/RNA hybridization assay}

For the detection of the miRNA-210 (sequence: 5'CUgugcGugugacAgCGgCUGA3', produced by IBIAN Technologies, Spain) a complementary DNA/RNA hybridization assay was employed. A complementary DNA capture probe containing a reactive thiol group which interacts with the gold surface (5'SH-T15-AGCCGCTGTCACACGCACA3', IBIAN Technologies, Spain) was designed. The probe also incorporated a spacer (sequence of 15 thymines) which keeps away the DNA strand from the sensor surface in order to facilitate the accessibility of the target miRNA. After thorough cleaning of the sensor chips with organic solvents (acetone and ethanol) and $\mathrm{O}_{2}$ plasma cleaning (100W, 45sccm, $2 \mathrm{~min}$ ), the sensor chips were placed in the experimental setup and a constant flow $\left(18 \mu \mathrm{L} \mathrm{min}^{-1}\right)$ of DEPC- $\mathrm{H}_{2} \mathrm{O}$ (Milli-Q water with $0.1 \%$ DEPC (diethyl pyrocarbonate)) was fixed. A solution of the DNA probe $(1 \mu \mathrm{M})$ prepared in PBS $50 \mathrm{mM}$ (containing 100 nM TCEP (tris(2-carboxyethyl) phosphine hydrochloride) to reduce eventual disulfide bonds formed between the thiol groups) was injected to achieve its direct immobilization on the surface of the nanogap antennas. Several solutions of miRNA-210 at different concentrations were prepared in SSC$5 X$ (sodium citrate buffer saline $75 \mathrm{mM}$ ) and sequentially injected into the sensor chip. A calibration curve was obtained and the LOD was determined by evaluating three times the value of the standard deviation of the $\Delta \lambda$ signal of a blank sample. After the injection of each of these samples, the surface was treated with a $5 \mathrm{mM} \mathrm{NaOH}$ solution $(60 \mathrm{~s}, 18 \mu \mathrm{L}$ $\min ^{-1}$,) to disrupt the DNA-RNA interactions, remove the RNA sequence and release the DNA capture probe for subsequent hybridization experiments. A reference non-complementary sequence, (miRNA-205 5'UCCUUCAUUCCACCGGAGUCUG3', 100nM, IBIAN Technologies, Spain) was additionally injected to evaluate the specificity of the sensor response to the target miRNA sequence.

\section{Results and Discussion}

Based on the colloidal hole-mask lithography process, we have produced large areas $\left(\approx 4.8 \mathrm{~cm}^{2}\right)$ of pseudo-ordered arrays of NPs on glass substrate chips. By tilting evaporation of the Ti sacrificial layer and relying on the remarkable anisotropy of the e-beam evaporation, we can precisely control the dimensions of the hole-mask. The tilted evaporation onto the NPs produce a "shadow" of the NPs, resulting in an ellipsoidal shaped hole-mask as observed in the SEM image in Figure 1a. The SEM image was obtained prior to the removal of the NPS, which are adhered onto the PMMA layer, and partially (top and left side) covered by the Ti deposition. A schematic that shows the possibility of tuning the long axis length of the ellipsoid mask $\left(L_{\text {mask }}\right)$ at the nanoscale level is depicted in Figure $1 \mathrm{~b}$. Considering the anisotropic evaporation angle $(\phi)$ of the $\mathrm{Ti}$, a complementary angle $(\theta)$ is formed between the surface of the PMMA layer and the tangent plane defined by the evaporated $\mathrm{Ti}$ (with a point of tangency at $\mathrm{T}$ formed by the radius of the sphere). Hence, it is possible to express $L_{\text {mask }}$ as a function of the angle $(\theta)$ and the NP radius $(r)$ as $L_{\text {mask }}=2 r / \sin (\theta$, which will ultimately define the length of the nanogap antennas.

Several combinations of tilted hole-mask angle and $\mathrm{Au}$ evaporation angle (Figure 1c) were evaluated. For an $80 \mathrm{~nm}$ diameter NPs, a $45^{\circ} \mathrm{Ti}$ evaporation produced a mask with an average length of $L_{\text {mask }} \approx 115 \mathrm{~nm}$. By using this mask, it is possible to produce nanogap antennas with arms ranging size between 50 to $60 \mathrm{~nm}$, which leads to a resonance spectrum in the optimum range for LSPR sensing. ${ }^{24}$ On the other hand, the size of the latex NPs determines and keeps constant the transversal axis of the arms forming the dimers. As can be observed in the SEM image in the Figure 1a, this method enables all nanogap antennas to be oriented in the same direction, which makes optimum the polarization of all the nanogap antennas using linearly polarized light. Once the mask is produced, the $\mathrm{O}_{2}$ plasma etches the PMMA layer underneath the $\mathrm{Ti}$, leaving the $\mathrm{SiO}_{2}$ surface accessible for deposition of the Au layer forming the dimers.

The optimal angle for the Au evaporation $\left(+/-10^{\circ}\right)$ was defined after observing the minimum tilting angle from which, approximately $90 \%$ of nanogaps were defined (gap size $>0$ $\mathrm{nm})$. Moreover, it is crucial for this fabrication method to have a precise control on the orientation of the long axis of the ellipsoidal mask respect to the edges of the glass chip. This fact is relevant for the geometrical conformation of the nanogap 
antennas and also determines the resonance spectrum for the polarization employed, as discussed in detail below. Figure 1d shows a SEM image of a sensor chip containing nanogap antennas fabricated on glass substrate using the conditions described above.
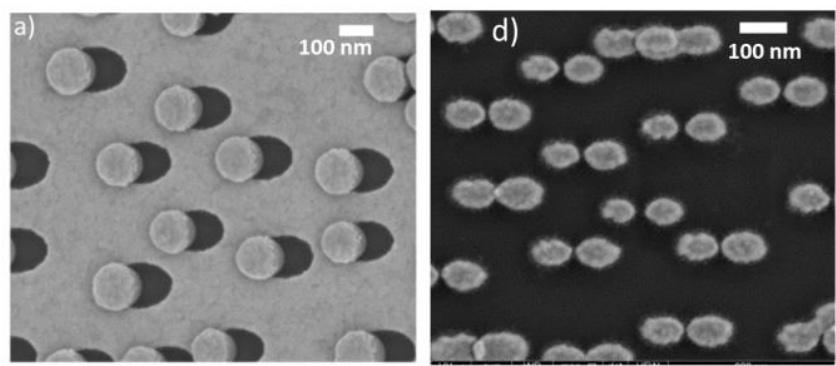

b)

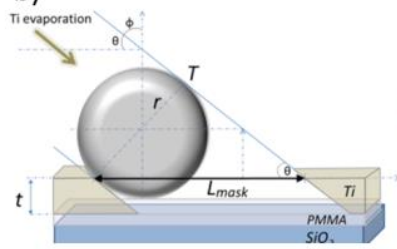

c)

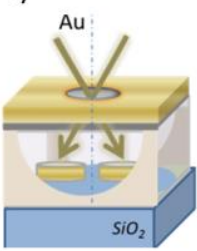

e)

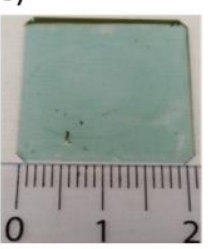

Fig. 1. Fabrication of nanogap antennas. a) SEM image of the elliptical mask obtained after tilted evaporation of $20 \mathrm{~nm}$ of Ti; b) Sketch of the flexibility for tuning the length of the long axis of the ellipsoid mask shape; c) Evaporation of gold using two opposite angles respect to the normal evaporation; d) SEM image of Au nanogap antennas over a glass substrate; e) Photograph of a fabricated sensor chip. A ruler $(\mathrm{mm})$ is shown as a reference scale.

The deposition of $19 \mathrm{~nm} \mathrm{Au}$ (each side) is performed as illustrated in Figure $1 \mathrm{c}$ at a rate of $0.05 \mathrm{~nm} / \mathrm{s}$. Prior to deposition of the Au forming the two arms of the nanogap antenna, a thin (1 $\mathrm{nm}) \mathrm{Ti}$ adhesion layer is added keeping constant the evaporation angles. Finally, a lift-off process exposes the Au nanogap antennas, a fact that can be visually observed for the bluish tone of the sensor chip. Figure 1e shows a photograph of a large nano-patterned substrate $(2.2 \mathrm{x}$ $2.2 \mathrm{~cm}^{2}$ ) sensor chip produced using the parameters detailed above.

SEM images were used to measure the gap size, the total length and the diameters of each arm of the antenna. Variations in the dimensions of the antennas arms and the gap size are observed, which are mainly attributed to the size dispersion of the latex NPs employed in the fabrication. Despite the differences observed in the dimensions and shape between nanogap antennas, the samples evaluated showed that nanogaps with average size of $11.6 \pm 4.7 \mathrm{~nm}$ were defined with a yield above $87 \%$. This fabrication strategy exhibits slightly lower resolution than other expensive and timeconsuming fabrication methods, such as e-beam lithography for gap sizes of the same range. ${ }^{19}$

FDTD simulations performed for these nanostructures were compared with discrete nanodisks (see Figure 2). Ideal nanogap antennas with a gap size equal to $15 \mathrm{~nm}$ onto a glass substrate shows a large confinement of a higher intensity EM field within the nanogap separating the two nanodisks (see Figure 2a), resulting on a higher sensitivity to changes of the RI as illustrated in Figure $2 \mathrm{~b}-\mathrm{d}$.

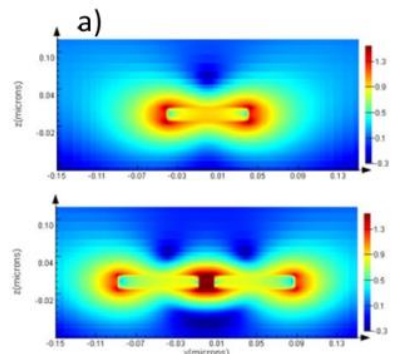

b)
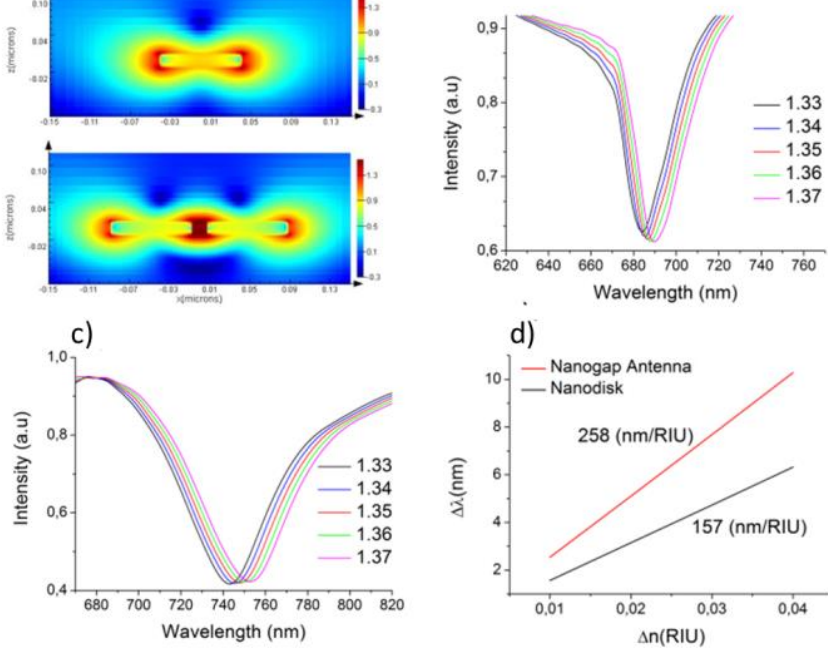

d)

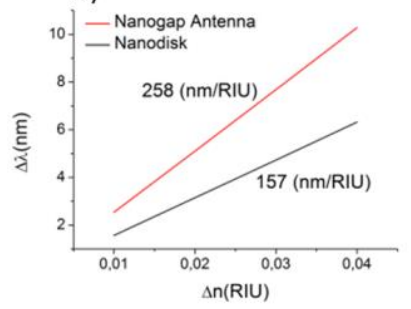

Fig. 2 a) Electric field profile of nanodisks (top) and nanogap antennas with a $15 \mathrm{~nm}$ gap (bottom) at their corresponding resonance wavelength. (b) FDTD numerical simulations for single nanodisks and (c) for nanogap antenna, showing the spectral response to RI changes from 1.33 to 1.37 ; d) Sensitivity to bulk RI changes for nanogap antennas and nanodisks.

Typically, optical characterization of LSPR nanostructures are performed by means of a dark field scattering spectroscopy setup where the extinction spectrum of nanostructures results in a peak-shaped spectral response. ${ }^{25}$ Our experimental characterization for bulk sensitivity and biosensing were conducted using an attenuated total internal reflection (ATR) configuration, which allows probing the sample with the evanescent plasmonic field. In this case the evanescent field strongly interacts with subwavelength features (i.e. the nanostructures) showing excited plasmonic resonance in the visible frequencies. ${ }^{18}$ Similarly to Kretschmann configuration, where signature from propagating plasmon resonance (SPP) can be observed on the reflected spectrum, we exploit this optical configuration to detect plasmonic internal reflection changes as sensed by the LSPR excited on the nanogap antennas. This optical configuration eliminates the constraints of transmission evaluation where the incident light has to go through the sample solution, where the microfluidic cell and the analyte sample can affect the measurements, and circumvents the complexity and the precision alignment required for dark field setups.

Figure 3a shows the schematic for the experimental setup. A collimated and TE polarized light is incident with a fixed angle of $80^{\circ}$ on the sensor chip using a trapezoidal prism. This particular incident angle has been previously reported to increase the sensitivity for a $6-7 \%$ surface nanodisks density. ${ }^{26}$ The reflected light is collected by a lens, coupling the light into 
an optical fiber, which is connected to the spectrophotometer An ellipsoidal incident light spot of approximately $1.5 \mathrm{~mm} \times 8.5$ $\mathrm{mm}$ (short and long radius, respectively) results in the excitation of a large number of nanogap antennas, which contribute to the overall signal. Due to the relative low-density $(\approx 7 \%)$ of the nanogap antennas on the glass substrate, nearfield interaction between them can be neglected.

a)

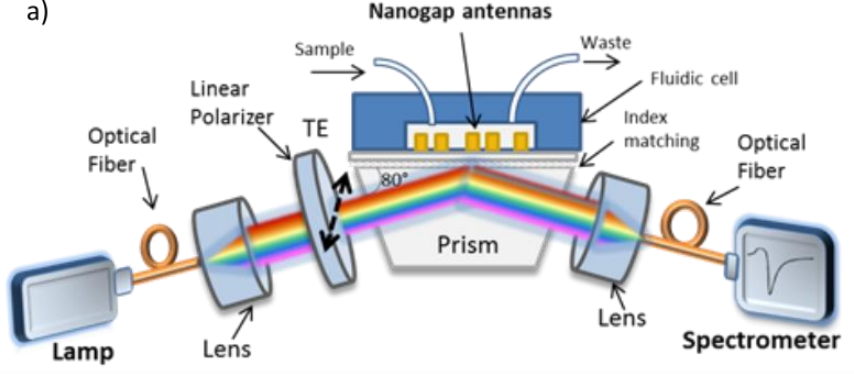

b)

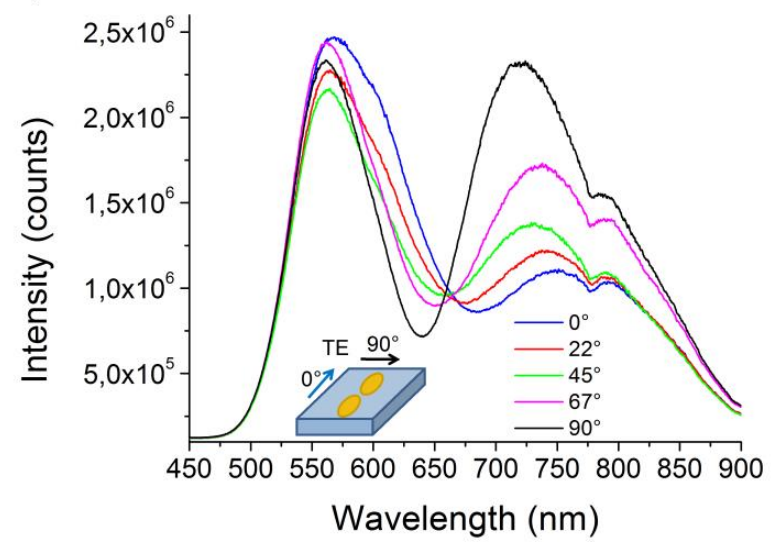

Fig. 3. Optical setup and optical characterization of the nanogap antennas. a) Schematic of the experimental setup. A fibered broadband light is collimated, linearly polarized (TE) and coupled with an $80^{\circ}$ trapezoidal prism. Reflectivity spectrum is measured through a fiber-coupled spectrophotometer. b) Spectral characterization of the nanogap antennas in a water environment for different orientations of incident TE polarized light with respect to the nanogap axis.

Experimental characterization of the produced nanogap antennas sensor chips was performed using the described optical setup using an in-plane (TE) linearly polarized light. A set of spectra for different angles of orientations of the nanogap respect to the electric field vector is presented in Figure 3b. Interestingly, the spectra showed a clear dependence of the orientation of the electric field respect to the axis of the nanogap. Presumably, the peak for the perpendicular $\left(90^{\circ}\right)$ nanogap orientation at $641 \mathrm{~nm}$ corresponds to the plasmon resonance excited in contiguous nanodisks with modes that weakly interact with each other, while for parallel nanogap orientation $\left(0^{\circ}\right)$ the peak at $685 \mathrm{~nm}$ corresponds to the excitation of the gap mode in pairs of nanodisks.

The change of the resonance peak location with the orientation can be exploited for tailoring the spectral response of these sensor chips. This tunability property of nanogap antennas excited under ATR configuration suggests the possibility of a "post-fabricated" fine-tuning, by rotating the sensor chip placed on the prism until the resonance peak is located on the intended position of the spectrum. This could be a desirable condition for surface enhanced spectroscopies, where intensity enhancement of the system can be drastically changed if the resonance peak matches the excitation or emission wavelengths to be amplified. However, it can be noticed an undesirable broadening of the resonance peak for the parallel orientation exciting the gap mode. We attribute this effect on the figure of merit to the large influence of gap size variations on the spectral peak position, which, in turn, is caused by the deviation of the nanofabrication process. This fabrication effect has a larger influence on the spectral response in the gap mode than the one observed for perpendicular orientation where the spectrum is dominated by the dimensions of single nanodisks. ${ }^{20}$ It has been reported that deviation on the nanofabrication process can hamper the figure of merit of LSPR sensing. ${ }^{19}$ As expected, the evaluation of the sensitivity for parallel and perpendicular configurations shows a different sensitivity to RI bulk changes $\left(\eta_{\mathrm{B}}\right)$. As can be noticed in Figure 4, there is a higher sensitivity for the TE polarized, parallel (422 nm/RIU) vs perpendicular (270 $\mathrm{nm} / \mathrm{RIU})$ ) orientation respect to the axis of the nanogap antennas. Interestingly, the sensitivity for the perpendicular nanogap orientation is very close to the $\eta_{B}$ obtained for single nanodisks (291 nm/RIU) of similar sizes to those forming the dimers, using identical experimental conditions. This result suggests that if the gap mode is not excited, the system with nanogap antennas behaves similarly to a sensor with individual Au nanodisks.

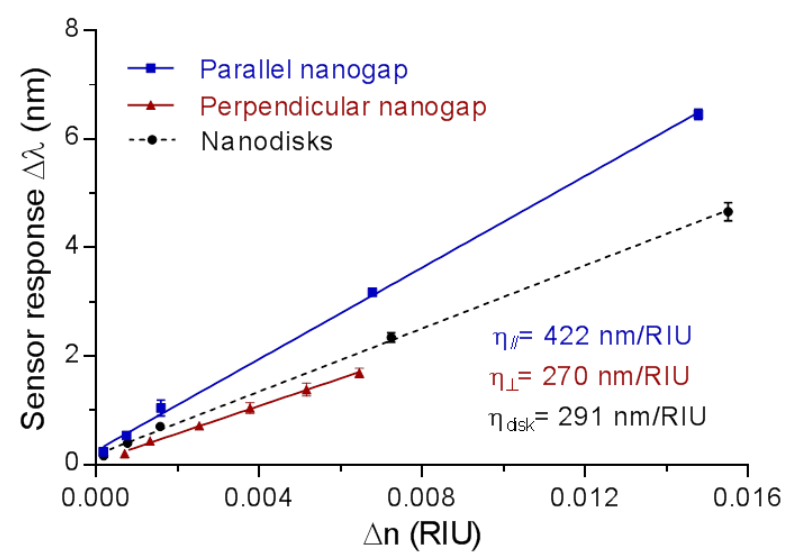

Fig. 4. Sensitivity to bulk RI changes using a set of glycerol solutions for perpendicular and parallel orientation of the nanogap respect to the TE polarized light. The bulk sensitivity of single nanodisks obtained under the same experimental conditions is also shown for comparison. 


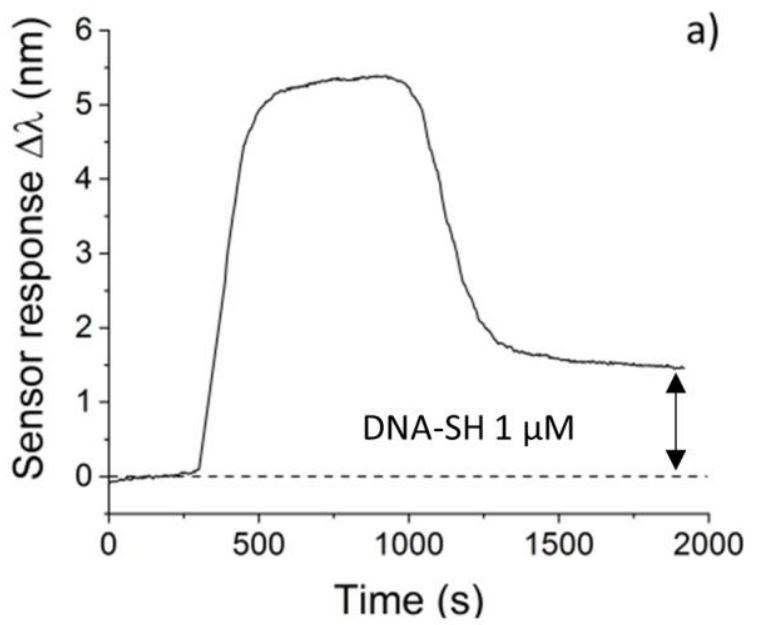

b)
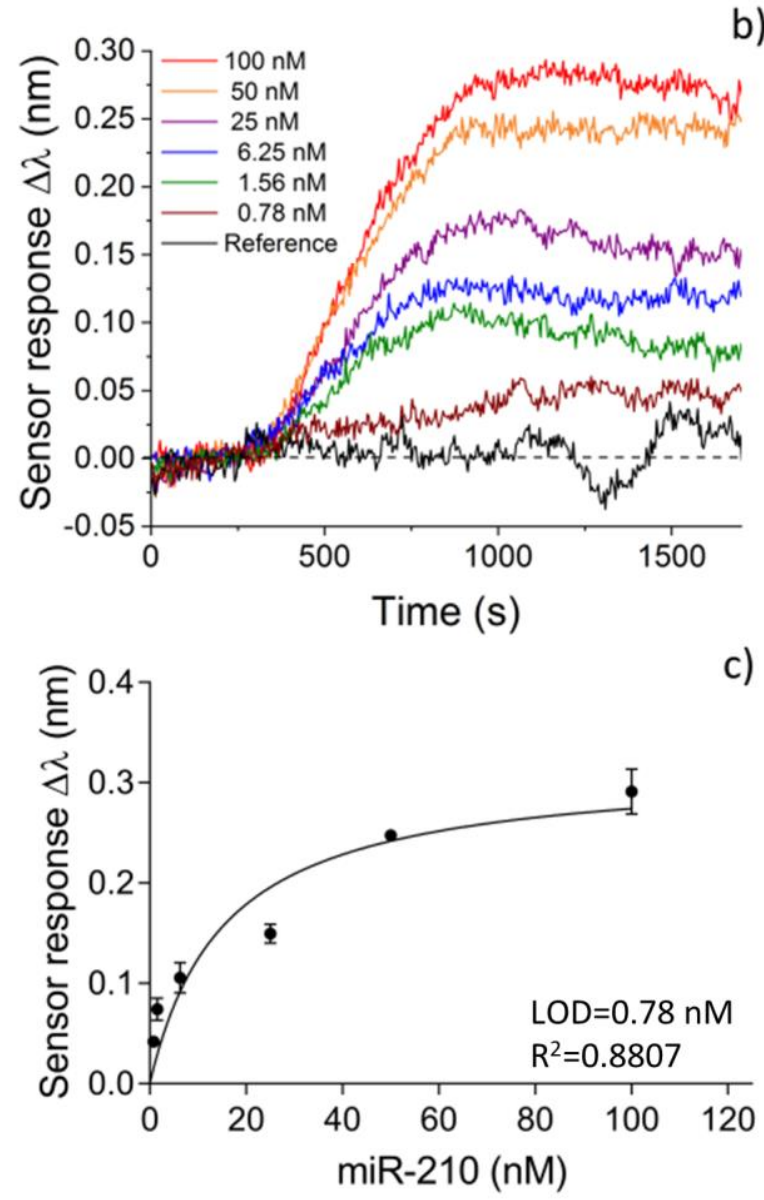

Figure 5. a) Dynamic monitoring of $\Delta \lambda$ during the in-situ immobilization of the SH-DNA probe $(1 \mu \mathrm{M})$ by the attachment of the thiol group to Au nanogap antennas. b) Sensorgrams obtained after injection of miR210 at different concentrations. A no complementary sequence (miRNA-205) injected at $100 \mathrm{nM}$ is also included (reference sensorgram in black). c) Calibration curve for miRNA-210, each point represents the mean \pm SD of 3 replicates.

The magnitude of changes observed on the sensitivity for the parallel and perpendicular orientation is much larger than the improvement attributed to the red-shift $(43 \mathrm{~nm})$ on the resonance wavelength. ${ }^{24,27}$ On the other hand, it should be noticed that there is consistency on the increasing trend of RI sensitivity between the numerical FDTD simulations and experimental results for the comparison of nanodisks and nanogap antennas (see Fig $2 \mathrm{~d}$ and Fig. 4). Although there are significant differences between the absolute values obtained theoretically, we attribute these disagreements to the excitation conditions used (normal illumination), since the convergence of the numerical models for this FDTD is limited for TIR illumination conditions.

Finally, in order to demonstrate the biosensing performance of the sensor chips, we selected the miRNA-210, a relevant biomarker for several types of cancer. Detection of miRNAs is challenging given their low expression, small size (20-24 nucleotides in length) and close similarity with other miRNAs. For the miRNA detection, the nanogap antennas are biofunctionalized with DNA capture probes that contain the complementary sequence to the target miRNA. The specific miRNA interacts with the immobilized capture probe and generates a change on the RI directly related to the miRNA concentration in the sample. Figure 5 a shows the dynamic response of the LSPR peak $\Delta \lambda$ during the injection of the DNA capture probe at a $1 \mu \mathrm{M}$ concentration. An initial baseline is defined at $0 \mathrm{~nm}$ as registered for the running buffer flow. Then a large response of about $5 \mathrm{~nm}$ is observed starting after $250 \mathrm{~s}$ and lasting approximately for $700 \mathrm{~s}$, corresponding to the bulk change sensed by the nanogap antennas. After about $750 \mathrm{~s}$ this value rapidly decreases as the microfluidic cell is filled again with the running buffer. The remaining difference of about $1.5 \mathrm{~nm}$ on $\Delta \lambda$ signal respect to the baseline corresponds to the total change of the RI in the surface of the nanogap antennas, induced by the immobilization of the DNA probe. Once the immobilization of the capture probe is achieved, sequential injection of miRNA-210 at different concentrations (0.78 nM, $1.56 \mathrm{nM}, 6.25 \mathrm{nM}, 25 \mathrm{nM}, 50 \mathrm{nM}, 100 \mathrm{nM}$ ) is conducted. After each hybridization event and prior to injecting the following concentration, the sensor surface is regenerated with a $\mathrm{NaOH}$ solution (as detailed in the experimental section), which allows the total dissociation of the miRNA-210 from the capture probe. Figure $5 b$ shows the change of the $\Delta \lambda$ signal for each concentration of miRNA-210. No changes on the $\Delta \lambda$ signal are observed after injection of a control sample corresponding to a non-complementary miRNA (miRNA-205) as can be also observed in this figure (black sensorgram). The calibration curve obtained from the set of miRNA-210 concentrations is shown in Figure 5c reaching an estimated LOD of $0.78 \mathrm{nM}$. It should be noticed that the signals obtained in Figure $5 b$ correspond to the direct detection of the target miRNA-210 with no need of further amplification steps. Most of published studies targeting the detection of miRNA have used different amplification strategies ${ }^{12}$ by means of proteins ${ }^{28,29}$ or enzymes. ${ }^{29}$ Our results have achieved a LOD $<1 \mathrm{nM}$ without any of these amplification steps, which is one order better than the LOD reported previously for miRNA-21012 prior the amplification steps. 
On the other hand, although for single nanostructure spectroscopy there are recent reports of sensitive detection of miRNA using nanospheres $(3 \mathrm{nM})^{30}$ or Au-nanocubes (pM range), ${ }^{31}$ our biosensor provides an alternative simple design not comprising complex optical read-out configurations, which make it a very suitable candidate for integration as a point of care testing device (POCT).

\section{Conclusions}

We have developed a method to produce a large-area of plasmonic nanogap antennas with interesting features for direct label-free biosensing. Nanogap antennas are fabricated using a hole-mask colloidal lithography process that allows customization of the geometry of the nanodisks and the nanogap separating them. Nanogap antennas were optically characterized using an ATR optical setup, where the plasmonic resonance showed the possibility of controlling their spectrum location by changing their orientation with respect to the TE polarized incident light. Higher bulk sensitivity was obtained for the parallel nanogap orientation (422 nm/RIU) vs the perpendicular orientation ( $278 \mathrm{~nm} / \mathrm{RIU})$. This result suggests that the improvement in performance is owed to the field enhancement generated by the gap effect. The biosensing performance of these sensor chips was demonstrated by the direct detection of a low molecular weight analyte, miRNA210 , reaching a LOD of only $0.78 \mathrm{nM}$. Based on the advantages that these nanostructures can offer (low fabrication cost and good performance), further development of nanogap antennas for the detection of multiple biomarkers has a promising future in the field of clinical biosensing.

\section{Conflicts of interest}

There are no conflicts of interest to declare.

\section{Acknowledgements}

We acknowledge the financial support from PreDICT project from the Spanish Research Agency (AEI), Ministry of Science, Innovation and Universities (TEC2016-78515-R). The ICN2 is funded by the CERCA programme / Generalitat de Catalunya. The ICN2 is supported by the Severo Ochoa Centres of Excellence programme, funded by AEI (grant no. SEV-20170706).

\section{Notes and references}

1. P. Gabriel, Nanobiophotonics, New York, McGraw-Hill Education, 2010.

2. Label-free biosensing: Advanced materials, devices and applications, 2018, Eds. M.J. Schöning and A. Poghossian, Springer, 2018

3. K.A. Willets and R.P. Van Duyne, Ann. Rev. Phys. Chem., 2007, 58, 267-297.
4. J. Homola, S.S. Yee and G. Gauglitz, Sens. Act. B, 1999, 54, 3-15

5. K.L. Kelly, E. Coronado, L.L. Zhao, G.C. Schatz, J. Phys. Chem. B., 2003, 170(3), 668-677.

6. H. Yockell-Lelièvre, F. Lussier, J.-F. Masson, J. Phys. Chem. C, 2015, 119, 28577-28585.

7. N. Pazos-Perez, L. Guerrini, R.A. Alvarez-Puebla, ACS Omega, 2018, 3, 17173-17179

8. W. Li, L. Zhang, J. Zhou and H. Wu, J. Mater. Chem. C, 2015, 3, 6479-6492

9. B. Sepúlveda, P.C. Angelomé, L.M. Lechuga and L.M. Liz-Marzán, Nano Today, 2009, 4, 244-251

10. A.B. Taylor and P. Zijlstra, ACS Sensors, 2017, 2, 1103-1122

11. D. Raghu, J. A. Christodoulides, M. Christophersen, J.L. Liu, G.P. Anderson, M. Robitaille, J.M. Byers and M.P. Raphael, Plos One, 2018, 13(8), e0202773

12. E. Mauriz, P. Dey and L.M. Lechuga, Analyst, 2019, 144, 7105-7129

13. P. Muehlschlegel, H.-J. Eisler, O.J. Martin, B. Hecht, D. Pohl, Science, 2005, 308, 1607-1609.

14. E. Prodan, C. Radloff, N.J. Halas and P. Nordlander, Science, 2003, 302, 419-422.

15. W. Zhang, L. Huang, C. Santschi and O.J. Martin, Nano Lett., 2010, 10, 1006-1011.

16. M. Righini, P. Ghenuche, S. Cherukulappurath, V. Myroshnychenko, F.J. García de Abajo and R. Quidant, Nano Lett., 2003, 9, 3387-3391.

17. A. Portela, T.a. Yano, C. Santschi, O.J. Martin, H. Tabata and M. Hara, J. Biophoton., 2017, 10, 294-302.

18. L. Novotny and N. van Hulst, Nat. Photon., 2010, 5, 83-90.

19. A. Portela, T. Yano, C. Santschi, H. Matsui, T. Hayashi, M. Hara, O.J.F. Martin and H. Tabata, Appl. Phys. Lett., 2014, 105, 091105.

20. H. Fischer and O.J.F. Martin, Opt. Expr., 2008 16, 9144-9154.

21. L. Tang, S.E. Kocabas, S. Latif, A.K. Okyay, D.-S. Ly-Gagnon, K.C. Saraswat and D.A. Miller, Nat. Photon., 2008, 2, 226229.

22. H. Fredriksson, Y. Alaverdyan, A. Dmitriev, C. Langhammer, D.S. Sutherland, M. Zaech and B. Kasemo, Adv. Mater., 2007, 19, 4297-4302.

23. P.B. Johnson and R.-W. Christy, Phys. Rev. B, 1972, 6, 4370.

24. M.A. Otte, B. Sepúlveda, W. Ni, J.P. Juste, L.M. Liz-Marzán, L.M. Lechuga, ACS Nano, 2010, 4, 349-357.

25. D.Y. Lei, A.I. Fernández-Domínguez, Y. Sonnefraud, K. Appavoo, R.F. Haglund Jr, J.B. Pendry and S.A. Maier, ACS Nano, 2012, 6, 1380-1386.

26. M. Soler, P. Mesa-Antunez, M.-C. Estevez, A.J. Ruiz-Sanchez, M.A. Otte, B. Sepulveda, D. Collado, C. Mayorga, M.J. Torres and E. Perez-Inestrosa, Biosens. Bioelectron., 2015, 66, 115123.

27. M.M. Miller and A.A. Lazarides, J. Phys. Chem. B, 2005, 109, 21556-21565.

28. X. Ding, Y. Yan, S. Li, Y. Zhang, W. Cheng, Q. Cheng and S. Ding, Anal. Chim. Acta, 2015, 874, 59-65. 


\section{ARTICLE}

29. S. Schmieder, J. Weißpflog, N. Danz, M. Hübner, S. Kreth, U. Klotzbach and F. Sonntag, Eng. Life Sci. 2017, 17, 1264-1270.

30. Y. Hu, L. Zhang, Y. Zhang, B. Wang, Y. Wang, Q. Fan, W. Huang and L. Wang, ACS Appl. Mater. Interf., 2015, 7, 24592466.

31. L. Zhang, J. Wang, J. Zhang, Y. Liu, L. Wu, J. Shen, Y. Zhang, Y. Hu, Q. Fan, W. Huang, L. Wang, ACS Sensors, 2017, 2, 14351440. 